\title{
Evaluation of the placebo effect in the trials of allergen immunotherapy effectiveness: meta-analysis of randomized and placebo-controlled trials
}

\author{
Michał Abramowicz, Jerzy Kruszewski, Andrzej Chciałowski \\ Department of Infectious Diseases and Allergology, Military Institute of Medicine, Warsaw, Poland \\ Adv Dermatol Allergol 2018; XXXV (6): 620-625 \\ DOI: https://doi.org/10.5114/ada.2018.77614
}

\begin{abstract}
Introduction: The total effect of the method of treatment is composed of its specific activity depending on its impact on the disease mechanism and the non-specific activity, i.e. the placebo effect. Many methods of treatment make use of such an inflammatory action.

Aim: To assess the placebo effect in the overall result of the specific immunotherapy and the analysis of its dependence on the type of specific immunotherapy, the disease, the age of a patient, the type of allergy, indicators used (objective and subjective), in patients with allergic diseases - asthma, allergic rhinitis and/or allergic conjunctivitis. Material and methods: A systematic review of Medline database was conducted using the EntrezPubmed search engine to find randomized placebo-controlled trials evaluating the effectiveness of specific immunotherapy in the treatment of asthma, allergic rhinitis and/or allergic conjunctivitis. After determining the contribution of the placebo effect for each of the test subjects, the calculation involved the average share of the placebo effect depending on the type of specific immunotherapy used, the type of disease, the age of the test subject, the type of allergy and the used measures of their effectiveness.

Results: The share of the placebo effect in the overall specific immunotherapy effect amounted to $39 \%$ and was comparable in the analyzed disease entities. A significantly higher share $(p<0.01)$ of $68 \%$ of the placebo effect in adult patients treated with sublingual immunotherapy vs. $29 \%$ in adult patients treated with subcutaneous immunotherapy.
\end{abstract}

Key words: immunotherapy, placebo effect, meta-analysis.

\section{Introduction}

Specific immunotherapy (SIT) is defined as the repeated administration of specific allergens to patients with IgE-mediated allergic disease in order to provide protection against the onset of symptoms of allergic and inflammatory reactions associated with natural exposure to allergens [1]. In 1911, Noon and Freeman [2-4] developed this method using one of Dunbar's concepts. Research on the effectiveness of SIT was initiated in 1954 by Frankland and Augustin who were the precursors of controlled clinical trials with placebo [5]. Due to their achievements, it was possible to document the value of the method and to classify SIT in the ranks of rational therapeutic medicine. We know today that these proceedings are not only more effective than placebo in reducing symptoms of diseases but they also inhibit the progression of diseases and new allergies [6]. In addi- tion, there are suggestions that the size of the placebo effect in this method is, however, lower than in the case of an allergic diseases pharmacotherapy [7]. But so far, our knowledge of the participation of the placebo effect in SIT has been negligible, because despite the existence of meta-analyses of the share of the placebo effect in allergic diseases and asthma, there have been no publications evaluating the share of the placebo effect in SIT [8-11].

\section{Aim}

The goal of this paper was to assess the extent of the placebo effect in the overall SIT effect and the analysis of its dependency on the type of SIT used, the type of disease, the age of the subject, the type of allergy (perennial and seasonal), measures used and their effectiveness

Address for correspondence: Michał Abramowicz MD, PhD, Department of Infectious Diseases and Allergology, Military Institute of Medicine, 128 Szaserów St, 04-141 Warsaw, Poland, phone: +48 2281029 09, e-mail: mabramowicz@wim.mil.pl Received: 18.04.2017, accepted: 12.11.2017. 
(objective and subjective indicators), in patients with the following allergic diseases - asthma, allergic rhinitis (AR) and/or allergic conjunctivitis (AC), in the published high quality studies on the effectiveness of immunotherapy.

\section{Material and methods}

A systematic review of Medline database was conducted using EntrezPubmed search engine for an initial search of the published randomized controlled trials evaluating the effectiveness of SIT in the treatment of AR and asthma by the following search criteria: subcutaneous immunotherapy (SCIT), sublingual immunotherapy (SLIT), randomized, placebo and controlled trials. Among the search results, 292 (100\%) publications met the above criteria.

Twenty-one (7\%) of them were included for further analysis (Table 1). The selection had to meet the following criteria: a given number of examined patients and their age, time, defined indicator evaluating the effectiveness of SIT allowing for the calculation of the effect in the placebo group and those subjected to immunotherapy, and the share of the placebo effect in the overall effect of treatment. The analysis concerned 1181 patients from clinical studies, of whom 623 were on SIT and 558 on placebo. An analysis was conducted regarding the size of the placebo effect and its relationship with the types of subjective indicators (asthma score, rhinitis score, drug score, drug consumption, upper and lower respiratory tract scales, and ocular VAS scale), objective ones (spirometry, peak expiratory flow rate $-\mathrm{PEFR}$ ) and depending on the SIT method: SCIT $(n=453)$ VS. SLIT $(n=728)$, type of allergy: seasonal $(n=545)$ vs. perennial $(n=757)$, disease entity: AR and/or AC $(n=735)$ vs. asthma ( $n=$ $808)$, and the patient's age: adults $(n=597)$ vs. children $(n=584)$.

Calculations were performed in collaboration with Assistant Professor Mieczyslaw Kłopotek, PhD. Eng. and Maciej Michalewicz, PhD. Eng. (Institute of Computer Science, Polish Academy of Sciences). For each study, wherever possible, three types of parameters were calculated: 1. (ED) the effect of the drug - which is a reflection of changes in the value of the examined parameter in the group receiving the drugs;

2. (EP) the placebo effect - which is a reflection of changes in the value of the parameter in the placebo group;

3. (SEP) share of the placebo effect - which reflects the percentage of the placebo effect in the total effect of treatment (TET).

ED - the effect of treatment - was understood as a percentage (or fractional) share of the improvement of a given index after the treatment of the total theoretically possible improvement (i.e. 100\% improvement means obtaining the state for formal or customary standard). A decrease or an increase in a given indicator was taken as an improvement in accordance with a medically accepted direction of improvement. Deterioration was treated as $E D=0$.

$E P$ - the placebo effect - was understood as a percentage (or fraction) of the index to improve the treatment of apparent total theoretically possible improvement (i.e. 100\% improvement means obtaining the state for formal or customary standard). An improvement means a decrease or an increase in a given indicator in accordance with medically accepted direction of improvement. Deterioration was treated as $E P=0$.

The SEP is the ratio of EP/ED, if it can be counted (when dispatching data on the EP and ED and ED >0), or the percentage or relatively fractional share of the improvement of a given indicator after apparent treatment in improvement during the real treatment (i.e. 100\% improvement is a state obtained in apparent treatment of such a condition, which has been attained in the real treatment). Deterioration was treated as EP $=0$.

The calculation of the first two indicators was not always possible. They could be counted when:

- the analyzed indicator was of an increasing or decreasing character with a known or obvious highest value (or lowest) or the value of the standard, as related to such indicators as asthma score, rhinitis score, drug score,

- the analyzed indicator was either ascending or descending and it was possible to assume its highest (or

Table 1. Studies included

\begin{aligned} & \hline 1 Eifan AO (Clin Exp Allergy 2010) \\ & \hline 2 Adkinson FA (N Engl J Med 1997) \\ & \hline 3 Zielen S (J Allergy Clin 2010) \\ & \hline 4 Ameal A (Allergy 2005) \\ & \hline 6 Creticos PS (N Engl J Med 1996) \\ & \hline 7 Durhan SR (N Engl J Med 1999) \\ & \hline 8 Tabar Al (Pediatr Allergy Immunol 2008) \\ & \hline 9 Marcucci F (Pediatr Allergy Immunol 2005) \\ & \hline 10 Hirsch T (Pediatr Allergy Immunol 1997) \\ & \hline 11 Pajno GB (Allergy 2000) \\ & \hline 12 Ippoliti F (Pediatr Allergy Immunol 2003) \\ & \hline 13 Bahceciler NN (Pediatr Pulmonol 2001) \\ & \hline 14 Rolinck-Werninghaus C (Allergy 2004) \\ & \hline 15 Pajno GB (Allergy 2004) \\ & \hline 16 Eifan AO (Clin Exp Allergy 2010) \\ & \hline 17 Tonnel AB (Allergy 2004) \\ & \hline 18 Ott H (Allergy 2009) \\ & \hline 19 Passalacqua G (Allergy 2006) \\ & \hline 20 Guez S (Allergy 2000) \\ & \hline 21 Bousquet J (Allergy 1999) \\ & \hline\end{aligned}


lowest) value or standard range, as related to such indicators as forced expiratory volume in $1 \mathrm{~s}\left(\mathrm{FEV}_{1}\right)$, PEFR, the level of drug consumption, worsening pulmonary, nasal and ocular symptoms.

The research was carried out by the so-called metaanalysis, considering a group of patients examined with one index in one publication as an analyzed object. The ED was determined for a subgroup of patients from this study, for whom the real treatment was conducted, while EP for the subgroup of patients for whom the apparent treatment was applied. Usually, the two sub-groups were comparable in terms of size. The SEP is a de facto indicator of the object of research, and not of an individual person. Both ED and EP were calculated based on the average values of the respective sub-groups before and after the treatment with respect to the relevant standards.

An assessment of the significance of the arithmetic averages of ED (i.e. those actively treated) and EP (i.e. those receiving placebo) for the objects of treatment using Student $t$-test for the so-called dependent groups was conducted. It was assumed that they differed from each other, if the significance level was below $5 \%$ or above $95 \%$. No change would mean that the effect of treatment is completely a placebo effect (SEP $=100 \%$ ).

Once SEP for each of the test objects was calculated, then SEP\% was calculated depending on the type of allergy (perennial, seasonal), disease entity (asthma, AR and/or AC), age (children, adults), and SIT method (SCIT, SLIT).

\section{Statistical analysis}

The results are illustrated graphically and in tabular form, showing average values and levels of significance of differences between different groups. The levels of sta- tistical significance for the differences were calculated using the Student $t$-test for independent groups. As before, it was assumed that they differed from each other, if the significance level was below $5 \%$ or above $95 \%$. No change would mean that the effect of treatment is completely a placebo effect $($ SEP $=100 \%)$.

\section{Results}

Based on the analysis of research publications on the effectiveness of SIT-controlled trials, it was demonstrated that the cumulative average SEP in the TET on the basis of all assessed indicators amounted to 39\%.

The highest SEP in the previously established parameters depending on disease, age, type of allergy (perennial and seasonal), used measures (indicators) of their effectiveness found in adults was 53\% and, in turn, regarding seasonal allergies $49 \%$, asthma $46 \%$, in subjective indicators 44\%, SCIT 42\%, AR and/or AC 39\%, SLIT $38 \%$, perennial allergy $35 \%$, children 33\%, and the lowest was (in line with our expectations) in objective indicators for assessing the effectiveness of SIT and amounted to $25 \%$ (Figure 1). In the analysis of SEP depending on a disease, age, type of allergy (perennial and seasonal), used measures (indicators) of their effectiveness despite mathematical differences, there was no statistical significance.

Figure 2 illustrates the average percentage of SEP in TET depending on age and the method used. A significantly higher share $(p<0.01)$ of more than $68 \%$ of the placebo effect was achieved in adults treated with SLIT compared to $29 \%$ with the SEP in adults treated with SCIT. There was no similar significance despite the mathematical difference in groups of children using SLIT vs. SCIT.

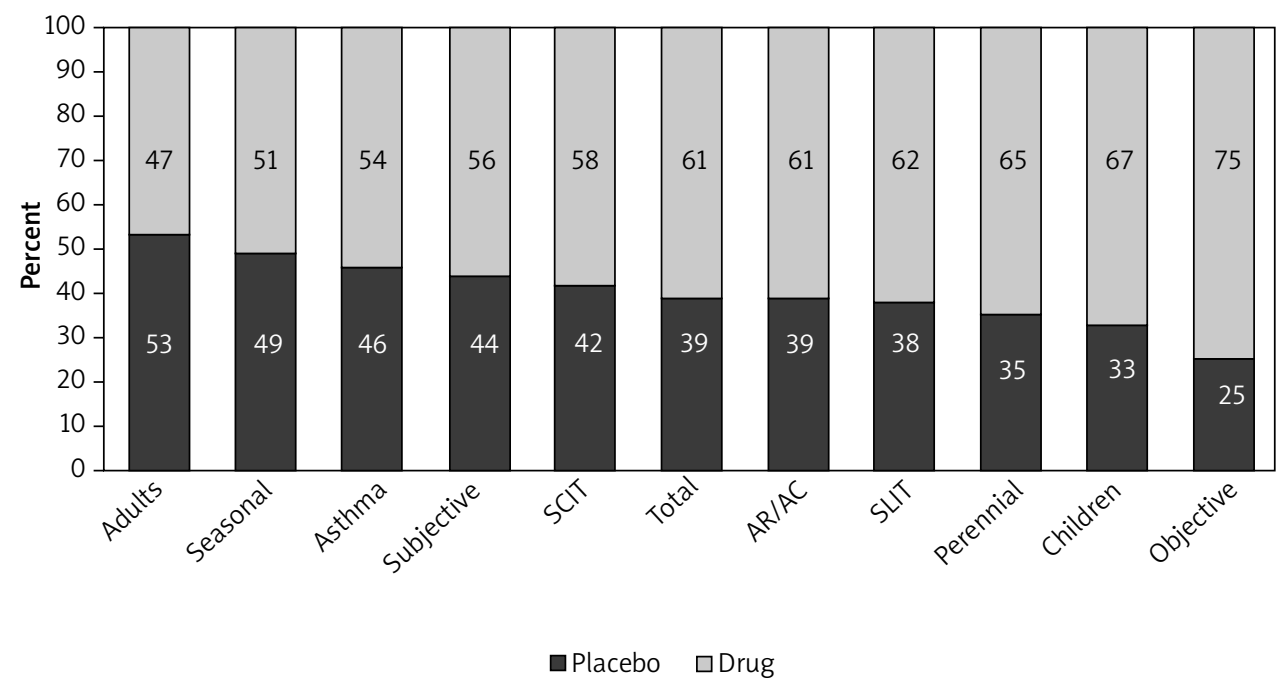

Figure 1. Average share of the placebo effect in the overall effect of treatment in placebo-controlled studies, the effectiveness of the SIT in all evaluated parameters dependent on the applied immunotherapy, disease entity, age, type of allergy (perennial and seasonal) and the used measures (indicators) of their effectiveness 


\section{Discussion}

The SIT calculated on the basis of studies included in the analysis of SEP in TET amounted to $39 \%$ on average. This size was comparable in both disease entities, i.e. asthma and AR and/or AC. These considerations show that patients with allergic diseases and indication for applying SIT, are very prone to the placebo effect and this effect was assessed and documented in the placebo-controlled clinical studies [7, 12]. A higher placebo effect - as high as $50 \%$ - is based on circumstantial evidence demonstrated by Wedi et al. [11]. This is probably because of a significant share of psychosomatic phenomena in shaping the image of their disease and its treatment [13]. In the evaluation of EP size, we are usually limited to its absolute value. On this basis, it is rightly believed to be particularly high in allergic diseases. On the basis of the quoted work and the current research, it seems that its proper and thorough evaluation in the clinical efficacy of drugs can only be performed in the context of the comparison taking into account the observed size of the TET in the actively treated group.

The analysis of selected factors that may affect the size of SEP showed that the most important dependence involved SCIT and SLIT in adults. SEP was particularly high in adults treated with SLIT (68\%) and differed significantly from adults undergoing SCIT treatment (29\%). Narkus et al. in their analysis presented different results, which showed an average placebo effect on the basis of SMS (symptom-medication score) - at 52\% for patients treated with SCIT and only $1 \%$ for those treated with SLIT [9].

Despite finding mathematical differences in other factors selected for the study such as the type of SIT regardless of age, type of allergy, used measures (indicators) of their effectiveness and the type of disease entity, the differences were not statistically significant, and so we can only talk about certain trends. It seems that the main reason for this situation was low cardinality of the groups of patients selected for the analysis.

In the current analysis, the total SEP for all studies amounted to $39 \%$ and was comparable to research on the second-generation antihistamines conducted by Radziwitt et al., who assessed SEP in studies of selected antihistamines applied in allergic diseases and asthma $[7,8]$. The SEP calculated in the current study in the case of SIT of $39 \%$ was higher compared to $29 \%$ SEP in the case of fluticasone, or $26 \%$ in the case of beclomethasone and even much higher in comparison to $17 \%$ SEP in the case of formoterol - drugs commonly used in the treatment of asthma. However, compared to the secondgeneration antihistamines such as cetirizine, loratadine or fexofenadine, SEP was $40 \%, 38 \%$ and $32 \%$, respectively, and was almost identical to the results of SEP in SIT in the present research paper. It is worth noting that the aforementioned authors also assessed the studies for the treatment of diseases other than allergic ones.

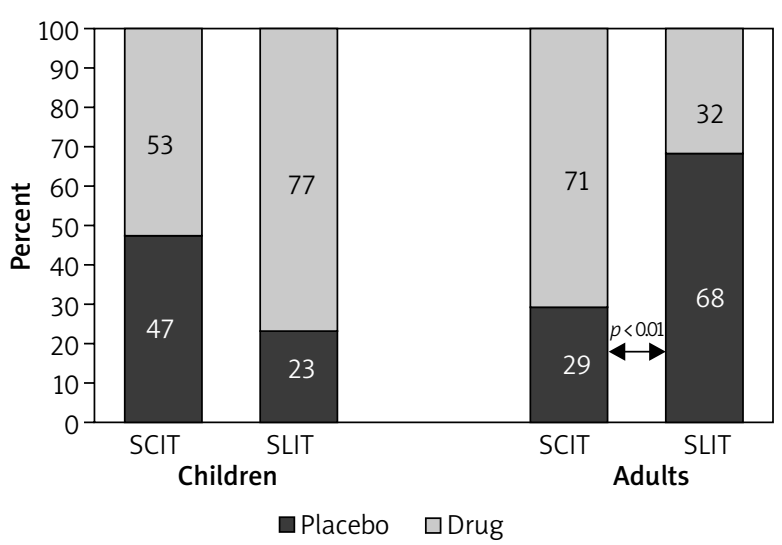

Figure 2. Average share of the placebo effect in the overall effect of treatment in placebo-controlled SIT efficacy studies depending on age and the method used

The SEP in the application of captopril in the treatment of hypertension amounted to only $17 \%$, which was comparable to SEP in the case of formoterol. It was significantly lower compared to the case of all evaluated drugs used in allergic diseases and asthma, as well as in relation to SIT in the analyzed study. This confirms that patients suffering from allergic diseases are more likely than patients with other somatoform disorders to be susceptible to the placebo effect, hence SEP in TET in these groups is high [7]. Overall, there were no large differences in the size of SEP between the two methods - SLIT and SCIT, which was caused by its completely different behavior in groups of children and adults. It is generally believed that the placebo effect in the treatment of children is lower than in adults, which was also confirmed by the trends observed in the present analysis [14]. However, an interesting finding is a particularly high SEP in adult subjects undergoing SLIT and its substantial difference in comparison to a group of children treated with SCIT (68 vs. 29\%). Despite convincing evidence that in certain indications, SLIT method is more effective than placebo, the relatively high SEP in its TET is worth some comment. It seems that this situation, in addition to the aforementioned varying susceptibility to EP among children and adults, may reflect differences characterizing both SIT methods; the differences important not only from the point of view of the patient, but also health policy. These differences may stem from the formation of different images of SLIT and SCIT value in terms of functioning in the perceptions of patients, which so far has not been taken into account in the evaluation of their effectiveness. SLIT method, implemented without injections, within the framework of self-treatment, relatively safe, does not require having to involve additional time and resources, advertised as a novelty, by most patients it can be seen as a more comfortable and friendly method than SCIT. Its important substantive defects, mainly due to general problems of self-medication and the higher price and the need for 
the daily admission of a vaccine, may be of a significant but rather secondary importance. Adverse reactions after SLIT in the form of itching of the throat, burning of the mucous membrane of the mouth, feeling of its edema, nasal itching, runny nose, sneezing, conjunctivitis redness, tearing, hoarseness, dry cough, pruritus or urticaria are usually not enough to surpass the very fear of injection, which is the basis of SCIT, which in some patients (despite a general belief that the injection is more effective than droplets or tablets) can dominate and be the reason for strong faith in a more effective mechanism of action and create a positive attitude to SLIT [15].

The lowest SEP was observed in patients who had objective indicators used in the assessment of the effectiveness of SIT, which confirms the expectations [7]. Unfortunately, the observed trend was the result of the analysis of only a small number of studies, mainly spirometry in patients with asthma, and which monitored various phenomena in laboratory studies during SIT, as mentioned later in the discussion. Although laboratory parameters, as devoid of subjective judgments, seem to be more effective than the clinical assessment, at this point it should be stressed that, despite a number of additional tests and a variety of further proposals, we still do not have a reliable indicator that could be used in assessing the effectiveness of SIT [16]. It is recognized that well-defined, objectified subjective indicators in the form of various scales of symptoms (pulmonary, nasal, ocular), frequency of visits, frequency and duration of hospitalization and inability to work, the frequency and number of medication, which are also important tools for the study of quality of life, allow for a reliable assessment of changes during treatment. Despite some inaccuracies of subjective indicators, their advantage is that they directly assess patients' sensation, are not always easy to apply, but take little time, are non-invasive and inexpensive. It happens that patients, despite the lack of significant improvement using objective indicators such as spirometry, show improvement on the scale of severity of "pulmonary symptoms", which is usually closer to the threshold of perception of improvement by patients, but remains subjective.

Among the analyzed publications, most concerned allergies in the course of all-year allergic sensitization, which translated into a greater number of such patients (almost 75\%) when compared to the course of allergic sensitization to seasonal allergens. It is not clear how SEP would behave in the comparison of both groups, if the two groups were similar in frequencies, so the observed trend would not exceed the limit of significance. The results of the high placebo effect are well known for patients desensitized against mite, who were subjected to SCIT. These results range between $24 \%$ and $41 \%$ compared to $6 \%$ and $25 \%$ for seasonal allergens [9].

The analysis of the type of indicators to assess the effectiveness of SIT concerned mainly the patients with asthma and their treatments, such as spirometry and PEFR. In the analyzed papers, simple objective tests were not used to assess the effectiveness of SIT in patients with AR/AC. In the course of defining and determining useful objective indicators, nobody took into account the concentration of IgE, aslgE, IL13, IgG4, the number of CD40 + B cells, due to their incidental application (e.g. in one study regarding 30 subjects), the lack of comprehensive source data and the absence of recognized standards for certain age. Therefore, the analysis of SEP in TET at patients with AR/AC was based solely on subjective indicators.

To sum up, the results presented in this paper regarding meta-analysis of randomized placebo-controlled efficacy studies of SIT confirmed the usefulness of the proposed indicators in assessing the value of this method in allergic diseases. Scarce literature data, which used similar ratios in relation to other treatments, however, give a better picture of SIT in relation to the pharmacotherapy of asthma and allergic diseases. A relatively high SEP in TET SIT has a different pronunciation when taking into account its values observed in clinical efficacy of antihistamines and anti-asthmatic drugs [7, 8]. The value of SIT as a method of proceeding in allergic diseases, which determines its active operation, is similar to the use of antihistamines, although treatments use different mechanisms of action, indications, methods of use and efficacy, and are used in different ranges, while the objective is always the same - reducing and relieving symptoms.

\section{Conclusions}

The study has led to the following conclusions: The share of the placebo effect in the overall SIT effect amounted to $39 \%$ and was comparable in the analyzed disease entities, i.e. asthma and AR and/or AC. The analysis of selected factors that may influence the size of the SEP during SIT allowed to identify only certain trends (except for a significant difference between the group of adults undergoing SLIT compared with SCIT) and indicates the existing differences in properties and real value of both SIT methods, hitherto little taken into account, but important from a practical point of view.

\section{Conflict of interest}

The authors declare no conflict of interest.

\section{References}

1. Joint Task Force on Practice Parameters; American Academy of Allergy, Asthma and Immunology; American College of Allergy, Asthma and Immunology; Joint Council of Allergy, Asthma and Immunology. Allergen immunotherapy: a practice parameter second update. J Allergy Clin Immunol 2007; 120: 25-85. 
2. Dunbar WP. The present state of our knowledge of hay-fever. J Hyg (Lond) 1913; 13: 105-48.

3. Noon L, Cantab BC. Prophylactic inoculation against hay fever. Lancet 1911; 177: 1572-3.

4. Freeman J. Further observations on the treatment of hay fever by hypodermic inoculations of pollen vaccine. Lancet 1911; 178: 814-7.

5. Frankland AW, Augustin R. Prophylaxis of summer hay-fever and asthma: a controlled trial comparing crude grass-pollen extracts with the isolated main protein component. Lancet 1954; 266: 1055-7.

6. Bousquet J, Khaltaev N, Cruzz AA, et al. Allergic Rhinitis and Its Impact on Asthma (ARIA 2008). Allergy 2008; 68: 8-160.

7. Radziwitł K, Kruszewski J. Evaluation of the size of the placebo effect in treatments of allergic diseases and asthma based on a meta-analysis of efficacy trials of drugs. Post Dermatol Alergol 2011; 28: 372-7.

8. Radziwitt K, Kruszewski J. Assessment of the size of the placebo effect in treating asthma based on meta-analysis of efficacy trials of selected antiasthmatic drugs. Pneumonol Alergol Pol 2012; 80: 301-7.

9. Narkus A, Lehnigk U, Haefner D, et al. The placebo effect in allergen-specific immunotherapy trials. Clin Transl Allergy 2013; 3: 42.

10. Dutile S, Kaptchuk TJ, Wechsler ME. The placebo effect in asthma. Curr Allergy Asthma Rep 2014; 14: 456.

11. Wedi B, Wieczorek D, Kapp A. Placebo effect in clinical trials with allergen-specific immunotherapy with inhalant allergens. Hautarzt 2017; 68: 297-306.

12. del Cuvillo A, Sastre J, Bartra J, et al. Placebo effect in clinical trials involving patients with allergic rhinitis. J Investig Allergol Clin Immunol 2011; 21 (Suppl 3): 40-5.

13. Zalewska-Janowska A. Psychodermatologia w alergologii. Alergia Astma Immunol 2010; 15: 109-17.

14. Kubik A, Skowronek-Bała B, Zajac A, Kaciński M. Placebo test in the diagnostics of pseudoepileptic seizures in children and adolescents. Przegl Lek 2004; 61: 1244-52.

15. De Craen AJ, Tijssen JG, De Gans J, Kleijnen J. Placebo effect in the acute treatment of migraine: subcutaneous placebos are better than oral placebos. J Neurol 2000; 247: 183-8.

16. Calderon MA, Eichel A, Makatsori M, Pfaar O. Comparability of subcutaneous and sublingual immunotherapy outcomes in allergic rhinitis clinical trials. Curr Opin Allergy Clin Immunol 2012; 12: 249-56. 